\title{
Graphs containing triangles are not 3-common
}

\author{
James Cummings* and Michael Young
}

\begin{abstract}
A finite graph $G$ is $k$-common if the minimum (over all $k$-colourings of the edges of $K_{n}$ ) of the number of monochromatic labelled copies of $G$ is asymptotically equal, as $n$ tends to infinity, to the expected number of such copies in a random $k$-colouring of the edges of $K_{n}$. Jagger, S̆ ك̆ovíček and Thomason showed that graphs which contain $K_{4}$ are not 2 -common. We prove that graphs which contain $K_{3}$ are not 3 -common.
\end{abstract}

AMS 2000 SUBJECT CLASSIFICATIONS: Primary 05D10; secondary 05C15. KEYWORDS AND PHRASES: Ramsey multiplicity, $k$-common graph.

\section{Introduction}

In this paper we prove a result about the Ramsey multiplicity constants of certain graphs, in the context of edge colourings in three colours. Before we state the result precisely we will give some background about Ramsey multiplicities.

A labelled copy of a graph $G$ in a graph $G^{\prime}$ is an injective function from $V(G)$ to $V\left(G^{\prime}\right)$ such that every edge of $G$ is mapped to an edge of $G^{\prime}$. Given a colouring of the edges of $G^{\prime}$, a monochromatic labelled copy of $G$ in $G^{\prime}$ is a labelled copy of $G$ in $G^{\prime}$ such that the images of the edges of $G$ all have the same colour.

If $G^{\prime}$ is complete, every injective map from $V(G)$ to $V\left(G^{\prime}\right)$ is a labelled copy of $G$ in $G^{\prime}$. We note that when $v(G)=t$ and $n \geq t$ there are $t$ ! $\left(\begin{array}{c}n \\ t\end{array}\right)$ labelled copies of $G$ in $K_{n}$, and that this quantity is asymptotic to $n^{t}$ as $n$ tends to infinity.

Let $G$ be a finite graph with $v(G)=t$ and let $k>1$. By Ramsey's theorem there is a natural number $C$ such that, for every $k$-colouring of the edges of $K_{n}$, among every set of $C$ vertices there is a monochromatic set of size $t$. It follows by a straightforward double counting argument that there is a constant $\epsilon>0$ such that, for every large enough $n$ and every $k$-colouring of the edges of $K_{n}$, there are at least $\epsilon n^{t}$ monochromatic labelled copies of $G$.

*The first author was partially supported by NSF Grants DMS-0400982 and DMS-0654046. 
This suggest that we should study the density of monochromatic labelled embeddings of $G$ in $K_{n}$, that is to say the proportion of labelled embeddings of $G$ in $K_{n}$ which are monochromatic.

Definition 1. Let $G$ be a graph with $v(G)=t$ and let $n \geq 1$. Let $H$ be a colouring of the edges of $K_{n}$.

1. $c_{\text {mon }}(G, H)$ is the number of labelled copies of $G$ in $K_{n}$ which are monochromatic for the colouring $H$.

2. $d_{m o n}(G, H)=\frac{c_{m o n}(G, H)}{t !\left(\begin{array}{c}n \\ t\end{array}\right)}$.

Definition 2. Let $G$ be a graph and let $k, n \geq 1$.

$C_{k}(G ; n)=\min \left\{d_{m o n}(G, H): H\right.$ is a $k$-colouring of the edges of $\left.K_{n}\right\}$.

The probabilistic method gives an easy upper bound on $C_{k}(G ; n)$. Let $v(G)=t$ and $e(G)=e$, and consider a random $k$-colouring $\mathbf{H}$ of the edges of $K_{n}$ in which the colours of the edges are independently chosen with equal probability. By linearity of expectation the expected value of $c_{m o n}(G, \mathbf{H})$ is $t !\left(\begin{array}{c}n \\ t\end{array}\right) k^{1-e}$, and we conclude that $C_{k}(G ; n) \leq k^{1-e}$.

In some cases this probabilistic bound is asymptotically tight. Goodman [3] gave an exact formula for the minimal number of monochromatic triangles in a 2-colouring of the edges of $K_{n}$. This quantity is asymptotic to $\frac{n^{3}}{24}$ as $n$ tends to infinity, so that $C_{2}\left(K_{3} ; n\right) \rightarrow \frac{1}{4}$. In this case $k=2$ and $e=3$, so that the probabilistic bound for $C_{2}\left(K_{3} ; n\right)$ is also $\frac{1}{4}$. Erdös [2] conjectured that $C_{2}(G ; n) \rightarrow 2^{1-e}$ for all complete $G$, and Burr and Rosta [1] conjectured that in fact this holds for all finite graphs $G$.

Thomason [7], showed that the conjecture by Erdős is false when $G=K_{4}$.

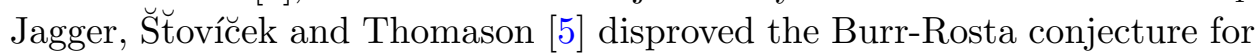
all graphs which contain $K_{4}$. Thomason [8] gave a simpler counterexample

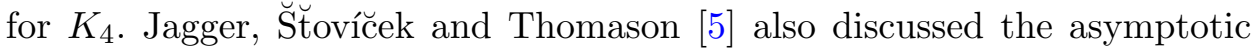
behaviour of $C_{k}(G ; n)$ for general values of $k$, and defined the notion of a $k$ common graph.

Fact 1. [5, section 3] Let $k \geq 1$ and let $G$ be a graph. $C_{k}(G ; n)$ increases with $n$.

Definition 3. Let $k \geq 1$ and let $G$ be a graph, with $e(G)=e$.

1. $C_{k}(G)=\lim _{n \rightarrow \infty} C_{k}(G ; n) . C_{k}(G)$ is the Ramsey multiplicity constant (for $k$ colours) of $G$.

2. $G$ is $k$-common if $C_{k}(G)=k^{1-e}$, and $k$-uncommon if $C_{k}(G)<k^{1-e}$. 
In this language the Burr-Rosta conjecture states that all graphs are 2common. Jagger, Štovíček and Thomason [5] and Jagger [4] proved several results about $k$-common and $k$-uncommon graphs.

1. If a graph $G$ contains $K_{4}$, then $G$ is not 2-common [5, theorem 12].

2. A notable conjecture by Sidorenko [6] states that if $G$ is a bipartite graph with $e(G)=e$, and $H$ is a graph with $v(H)=n$ and average degree $p n$, then the probability that a random map from $V(G)$ to $V(H)$ is a graph homomorphism is at least $p^{e}$. This conjecture has been established for several classes of bipartite graphs including complete bipartite graphs, trees and even cycles.

If a bipartite graph $G$ satisfies Sidorenko's conjecture, then $G$ is $k$ common for all $k$ [5, section 5].

3. If a graph $G$ is $k$-uncommon, then $G$ is $(k+1)$-uncommon [5, theorem 13]

4. If $G$ is a non-bipartite graph with $v(G)=t$, then $G$ is $k$-uncommon when $2^{2 k-2} \geq k^{t}[5$, theorem 14]. Hence $G$ is $k$-uncommon for all sufficiently large values of $k$.

5. Odd cycles are 3-uncommon [4].

Our main result is Theorem 1, which states that any graph containing $K_{3}$ is 3 -uncommon. The argument is algebraic and parallels that of $[5$, section 4]. In Section 2 we give a formula estimating the quantity $c_{m o n}(G, H)$. In Section 3 we define a notion of tensor product for $k$-colourings, and give a criterion for a graph to be $k$-uncommon. In Section 4 we prove Theorem 1 . Finally in Section 5 we record some questions and remarks.

\section{Counting monochromatic copies}

In this section we give an estimate for $c_{m o n}(G, H)$, the number of monochromatic labelled copies of $G$ in a colouring $H$ of the edges of $K_{n}$. We are most interested in the case of 3-colourings, but the analysis works for any number $k$ of colours. For the rest of this section we will fix some value $k$ with $k>1$, and will consider $k$-colourings for which the set of colours is $\{0, \ldots, k-1\}$. We will also fix a graph $G$ with $v(G)=t$ and $e(G)=e$.

Let $\zeta_{k}=\exp \left(\frac{2 \pi \imath}{k}\right)$, so that $\zeta_{k}$ is a primitive $k^{\text {th }}$ root of 1 . The $k$ complex $k^{\text {th }}$ roots of 1 are of the form $\zeta_{k}^{i}$ for $0 \leq i<k$. To lighten the notation we let $\zeta=\zeta_{k}$ for the rest of this section.

Let $p(x)=\sum_{j=0}^{k-1} x^{j}$. A trivial calculation shows that

$$
p\left(\zeta^{i}\right)=\left\{\begin{array}{lll}
k & \text { if } i \equiv 0 \quad \bmod k \\
0 & \text { if } i \neq \equiv 0 & \bmod k .
\end{array}\right.
$$


Officially a $k$-colouring of the edges of a complete graph with vertex set $V$ is a function $H$ from $[V]^{2}$ to $\{0, \ldots, k-1\}$. It will often be convenient to consider $H$ as a symmetric function from $V^{2}$ to $\{0, \ldots, k-1\}$, and to make the convention that $H(v, v)=0$ for all $v \in V$.

Definition 4. Let $H$ be a $k$-colouring of the edges of $K_{n}$. $A_{H}$ is the complexvalued function with domain $V\left(K_{n}\right)^{2}$ given by the formula $A_{H}(u, v)=\zeta^{H(u, v)}$.

We note that by our conventions $A_{H}(u, u)=1$ for all $u$.

Definition 5. Let $n>0$ and let $H$ be a $k$-colouring of the edges of $K_{n}$.

1. $F^{*}(G, H)$ is the set of $f: V(G) \rightarrow V\left(K_{n}\right)$ such that for some $i$ with $0 \leq i<k, A_{H}(f(u), f(v))=\zeta^{i}$ for every edge $u v \in E(G)$.

2. $I(G, H)$ is the set of $f \in F^{*}(G, H)$ which are injective.

It follows immediately from the definitions that $I(G, H)$ is the set of labelled copies of $G$ in $K_{n}$ which are monochromatic for $H$. By definition, $c_{\text {mon }}(G, H)=|I(G, H)|$ and $I(G, H) \subseteq F^{*}(G, H)$.

We now argue that $\left|F^{*}(G, H)\right|$ is a reasonably good approximation to $|I(G, H)|$ when $n$ is large. The elements of $F^{*}(G, H) \backslash I(G, H)$ are noninjective functions from $V(G)$ to $V\left(K_{n}\right)$, and the number of such functions is $n^{t}-t !\left(\begin{array}{c}n \\ t\end{array}\right)$, so $\left|F^{*}(G, H)\right| \backslash|I(G, H)|$ is $O\left(n^{t-1}\right)$. As we mentioned in the introduction, it follows from Ramsey's theorem that $|I(G, H)|$ is $\Theta\left(n^{t}\right)$.

For the rest of this section we fix the value of $n$, and we also fix a $k$-colouring $H$ of the edges of $K_{n}$. We will find an exact formula for $\left|F^{*}(G, H)\right|$, and in the following section we will exploit this formula and the fact that $c_{\text {mon }}(G, H)$ is close to $\left|F^{*}(G, H)\right|$.

Definition 6. Let $f: V(G) \rightarrow V\left(K_{n}\right)$ and $0 \leq i<k$.

1. $P(i, f)=\prod_{u v \in E(G)} p\left(\zeta^{-i} A_{H}(f(u), f(v))\right)$.

2. $Q(f)=\sum_{i=0}^{k-1} P(i, f)$.

Lemma 1. $\left|F^{*}(G, H)\right|=k^{-e} \sum_{f: V(G) \rightarrow V\left(K_{n}\right)} Q(f)$.

Proof. Fix $f: V(G) \rightarrow V\left(K_{n}\right)$. By the discussion of the properties of $\zeta$ and $p(x)$ at the start of this section, if $A_{H}(f(u), f(v))=\zeta^{i}$ for all $u v \in E(G)$ then $P(i, f)=k^{e}$; otherwise $P(i, f)=0$. It follows that if $f \in F^{*}(G, H)$ then $P(i, f)=k^{e}$ for one value of $i$ and $P(i, f)=0$ for all other values, while if $f \notin$ $F^{*}(G, H)$ then $P(i, f)=0$ for all $i$. Therefore $Q(f)=k^{e}$ if $f \in F^{*}(G, H)$, and $Q(f)=0$ if $f \notin F^{*}(G, H)$. Summing over all $f: V(G) \rightarrow V\left(K_{n}\right)$, we conclude that $\sum_{f: V(G) \rightarrow V\left(K_{n}\right)} Q(f)=k^{e}\left|F^{*}(G, H)\right|$. 
Expanding the product $P(i, f)$, we find that

$$
\begin{aligned}
P(i, f) & =\sum_{h: E(G) \rightarrow\{0,1, \ldots, k-1\}} \prod_{u v \in E(G)} \zeta^{-i h(u v)} A_{H}(f(u), f(v))^{h(u v)} \\
& =\sum_{h: E(G) \rightarrow\{0,1, \ldots, k-1\}} \zeta^{-i \sum_{u v \in E(G)} h(u v)} \prod_{u v \in E(G)} A_{H}(f(u), f(v))^{h(u v) .}
\end{aligned}
$$

Summing over $i$ and recalling the definition of $p(x)$,

$$
Q(f)=\sum_{h: E(G) \rightarrow\{0,1, \ldots, k-1\}} p\left(\zeta^{-\sum_{u v \in E(G)} h(u v)}\right) \prod_{u v \in E(G)} A_{H}(f(u), f(v))^{h(u v)} .
$$

The term $p\left(\zeta^{-\sum_{u v \in E(G)} h(u v)}\right)$ has the value $k$ when $\sum_{u v \in E(G)} h(u v) \equiv 0$ $\bmod k$, and the value zero when $\sum_{u v \in E(G)} h(u v) \not \equiv 0 \bmod k$. This motivates the following definition.

Definition 7. A $k$-colouring $h$ of the edges of $G$ is balanced if and only if $\sum_{u v \in E(G)} h(u v) \equiv 0 \bmod k . B_{k}(G)$ is the set of balanced $k$-colourings of the edges of $G$.

It follows that

$$
Q(f)=k \sum_{h \in B_{k}(G)} \prod_{u v \in E(G)} A_{H}(f(u), f(v))^{h(u v)},
$$

and so by Lemma 1 that

$$
\left|F^{*}(G, H)\right|=k^{1-e} \sum_{f: V(G) \rightarrow V\left(K_{n}\right), h \in B_{k}(G)} \prod_{u v \in E(G)} A_{H}(f(u), f(v))^{h(u v)} .
$$

Now we write the results of this calculation of $\left|F^{*}(G, H)\right|$ in a more palatable form.

Definition 8. Let $h$ be a $k$-colouring of the edges of $G$.

$$
\rho(h, H)=n^{-t} \sum_{f: V(G) \rightarrow V\left(K_{n}\right)} \prod_{u v \in E(G)} A_{H}(f(u), f(v))^{h(u v)} .
$$

We note that the quantity $\rho(h, H)$ is the average value of the product $\prod_{u v \in E(G)} A_{H}(f(u), f(v))^{h(u v)}$ taken over all $f: V(G) \rightarrow V\left(K_{n}\right)$.

Definition 9. $\Psi(G, H)=\sum_{h \in B_{k}(G)} \rho(h, H)$.

We now have a suggestive formula for $\left|F^{*}(G, H)\right|$. 
Lemma 2. $\left|F^{*}(G, H)\right|=k^{1-e} n^{t} \Psi(G, H)$.

We will see in the next section that $G$ is $k$-uncommon if and only if there exist $n$ and a $k$-colouring $H$ of the edges of $K_{n}$ with $\Psi(G, H)<1$.

Remark 1. The quantities $\rho(h, H)$ are complex numbers which may have nonzero imaginary parts or irrational real parts. They are algebraic numbers lying in the number field $\mathbb{Q}(\zeta)$. When we form the sum $\Psi(G, H)$ the result will always be a rational number.

Remark 2. When $k=2$ these formulae reduce to those of [5, section 4]. In particular the set of balanced 2-colourings corresponds to the set $\operatorname{ESSUB}(G)$ of even spanning subgraphs appearing in the formulae of [5].

Remark 3. We could use similar ideas to count copies of coloured graphs in $H$.

\section{A criterion for a graph to be $k$-common}

In this section we will define a product construction for $k$-colourings of the edges of complete graphs, and use this construction to relate the quantity $\Psi(G, H)$ defined in the preceding section to the question whether $G$ is $k$-common. The calculations here are along the same lines as those of $[8$, section 4].

Before defining the product construction, we recall our convention that $k$-colouring of the edges of a complete graph with vertex set $V$ can be regarded as a symmetric function $H$ from $V^{2}$ to $\{0, \ldots, k-1\}$ with $H(v, v)=0$ for all $v \in V$.

Definition 10. Let $G_{1}$ and $G_{2}$ be complete graphs. Let $V_{i}=V\left(G_{i}\right)$ and $H_{i}$ be a $k$-colouring of the edges of $V_{i}$ for $i=1,2 . H_{1} \otimes H_{2}$ is a $k$-colouring of the edges of the complete graph with vertex set $V_{1} \times V_{2}$, whose values are given by

$$
H_{1} \otimes H_{2}\left(\left(u_{1}, u_{2}\right),\left(v_{1}, v_{2}\right)\right) \equiv H_{1}\left(u_{1}, v_{1}\right)+H_{2}\left(u_{2}, v_{2}\right) \quad \bmod k
$$

for all $u_{1}, v_{1} \in V_{1}$ and $u_{2}, v_{2} \in V_{2}$.

We observe that

$$
A_{H_{1} \otimes H_{2}}\left(\left(u_{1}, u_{2}\right),\left(v_{1}, v_{2}\right)\right)=A_{H_{1}}\left(u_{1}, v_{1}\right) A_{H_{2}}\left(u_{2}, v_{2}\right) .
$$

Lemma 3. Let $G$ be a graph and $h$ be a mapping from $E(G)$ to $\{0,1, \ldots$, $k-1\}$. Let $\bar{K}_{m}$ be the colouring of the edges of $K_{m}$ such that all of the edges have colour 0 . Let $H_{1}$ be a k-colouring of the edges of $K_{n}$ and $H_{2}$ be a k-colouring of the edges of $K_{m}$. 
1. $\rho\left(h, H_{1} \otimes H_{2}\right)=\rho\left(h, H_{1}\right) \rho\left(h, H_{2}\right)$.

2. If $h(u v)=0$ for all $u v \in E(G)$, then $\rho\left(h, H_{1}\right)=1$.

3. $\rho\left(h, \bar{K}_{m}\right)=1$.

4. $\Psi\left(G, H_{1} \otimes \bar{K}_{m}\right)=\Psi\left(G, H_{1}\right)$.

The proof of Lemma 3 is a routine calculation. We note that if $H$ is a $k$-colouring of the edges of $K_{n}$, then $H \otimes \bar{K}_{m}$ can be described in a very simple way; each vertex $v$ of $K_{n}$ is replaced by a block of vertices $S_{v}$ with size $m$, a complete graph is formed whose vertex set is the union of these blocks, and every edge which joins a member of $S_{v}$ to a member of $S_{w}$ is given colour $H(v, w)$.

Lemma 4. Let $G$ be a graph with $e(G)=e$ and let $H$ be a $k$-colouring of the edges of $K_{n}$. Then $C_{k}(G) \leq k^{1-e} \Psi(G, H)$.

Proof. Consider the sequence of $k$-colourings $H_{m}=H \otimes \bar{K}_{m}$, where by definition $H_{m}$ is a $k$-colouring of the edges of $K_{n m}$. Since

$$
c_{\text {mon }}\left(G, H_{m}\right)=\left|I\left(G, H_{m}\right)\right| \leq\left|F^{*}\left(G, H_{m}\right)\right|=k^{1-e}(n m)^{t} \Psi\left(G, H_{m}\right)
$$

by Lemma 2 , and $\Psi(G, H)=\Psi\left(G, H_{m}\right)$ by Lemma 3 , we see that

$$
\frac{c_{m o n}\left(G, H_{m}\right)}{t !\left(\begin{array}{c}
n m \\
t
\end{array}\right)} \leq \frac{k^{1-e}(n m)^{t} \Psi(G, H)}{t !\left(\begin{array}{c}
n m \\
t
\end{array}\right)} .
$$

As $m$ tends to $\infty, \frac{(n m)^{t}}{t !\left(\begin{array}{c}n m \\ t\end{array}\right)}$ tends to 1 . So by taking the limit in the above inequality, $C_{k}(G) \leq k^{1-e} \Psi(G, H)$.

Lemma 4 implies that if $\Psi(G, H)<1$ for some $H$ then $G$ is not $k$-common, which is all we will need for the proof of Theorem 1. It is an interesting fact that the converse implication also holds.

Lemma 5. Let $G$ be a graph and let $k>1$. The following are equivalent.

1. G is k-uncommon.

2. There exist $n$ and a $k$-colouring $H$ of the edges of $K_{n}$ such that $\Psi(G$, $H)<1$.

Proof. Let $G$ be a graph with $v(G)=t$ and $e(G)=e$. If there is $H$ such that $\Psi(G, H)<1$, then by Lemma $4 C_{k}(G)<k^{1-e}$ and $G$ is $k$-uncommon.

Conversely suppose that $G$ is $k$-uncommon. By definition we have $C_{k}(G)<k^{1-e}$, and we let $\epsilon=k^{1-e}-C_{k}(G)$. Then $\epsilon>0$, and since $C_{k}(G ; n)$ increases with $n$ and has limit $C_{k}(G)$ we have $C_{k}(G ; n) \leq k^{1-e}-\epsilon$ for all $n$. 
We recall from Section 2 that for any $n$ and any $k$-colouring $H$ of the edges of $K_{n}, I(G, H) \subseteq F^{*}(G, H)$ and $\left|F^{*}(G, H) \backslash I(G, H)\right| \leq n^{t}-t !\left(\begin{array}{c}n \\ t\end{array}\right)$. The polynomial $n^{t}-t !\left(\begin{array}{l}n \\ t\end{array}\right)$ has degree $t-1$, so we may choose $n$ so large that $n^{t}-t !\left(\begin{array}{l}n \\ t\end{array}\right)<\epsilon n^{t}$.

By definition $C_{k}(G ; n)$ is the minimum of $\frac{|I(G, H)|}{t !\left(\begin{array}{c}n \\ t\end{array}\right)}$ over all $k$-colourings $H$ of the edges of $K_{n}$, so we may choose such an $H$ with $C_{k}(G ; n)=\frac{|I(G, H)|}{t !\left(\begin{array}{l}n \\ t\end{array}\right)}$. Then

$$
|I(G, H)| \leq\left(k^{1-e}-\epsilon\right) t !\left(\begin{array}{l}
n \\
t
\end{array}\right) \leq\left(k^{1-e}-\epsilon\right) n^{t}
$$

and

$$
\left|F^{*}(G, H)\right|-|I(G, H)| \leq n^{t}-t !\left(\begin{array}{c}
n \\
t
\end{array}\right)<\epsilon n^{t}
$$

so that

$$
\left|F^{*}(G, H)\right|<k^{1-e} n^{t} .
$$

It follows from Lemma 2 that $\Psi(G, H)<1$.

Remark 4. Lemma 5 implies that the property of being $k$-common is computably enumerable. That is to say there is an algorithm which takes $G$ as its input, and eventually halts if and only if $G$ is not $k$-common. The algorithm is easy to describe: enumerate all possible colourings, compute $\Psi(G, H)$ for each such colouring $H$, and halt if we find an $H$ such that $\Psi(G, H)<1$. It is not clear that the property of being $k$-common is computable, that is that there is an algorithm which halts on every $G$ and tells us whether $G$ is $k$-common or not.

\section{Graphs containing $K_{3}$}

In this section we prove our main result, that every graph which contains a triangle is 3-uncommon. Our approach is similar to that used in [5], and involves quadratic forms over finite fields. We use the field $G F(3)$ where $G F(2)$ is used in [5]. Compared with $G F(2)$, the field $G F(3)$ has the advantage that every quadratic form is diagonalizable, but the disadvantage that a diagonal matrix can have both +1 and -1 as entries on the diagonal.

We will consider the field $G F(3)$ as the set $\{0,1,2\}$ with the operations of addition and multiplication modulo 3 . As in Section 2 we will be doing computations with a complex root of unity, in this case the primitive third root $\zeta_{3}=\exp \left(\frac{2 \pi \imath}{3}\right)=\frac{-1+\imath \sqrt{3}}{2}$. We note that if $a, b \in G F(3)$ then $\zeta_{3}^{a} \zeta_{3}^{b}=\zeta_{3}^{a+b}$ and $\left(\zeta_{3}^{a}\right)^{b}=\zeta_{3}^{a b}$, where the sum $a+b$ and the product $a b$ are computed in $G F(3)$. 
Let $s$ be a positive integer and let $m=4 s+2$. We will construct a 3 -colouring of the edges of $K_{n}$ where $n=3^{m}$, starting from a certain quadratic form $q$ with coefficients in $G F(3)$.

$$
q=x_{1}^{2}+x_{2}^{2}+\cdots+x_{2 s+2}^{2}-x_{2 s+3}^{2}-x_{2 s+4}^{2}-\cdots-x_{4 s+2}^{2} .
$$

Now let $J_{s}$ be the following 3-colouring of the edges of a complete graph with $n$ vertices: the vertex set is $G F(3)^{m}$, the set of colours is $G F(3)$, and the colour of edge $x y$ is $q(x-y)$. It is clear that $q(x-y)=q(y-x)$, so that the colouring $J_{s}$ is well-defined.

Let $G$ be a graph with $V(G)=\{1,2 \ldots t\}$ and let $h$ be a 3-colouring of the edges of $G$, where we consider the colouring $h$ as taking values in $G F(3)$. Define $M(h)$ as the $t \times t$ matrix with entries from $G F(3)$ given by

$$
M(h)_{a b}= \begin{cases}h(a b) & \text { if } a b \in E(G), \\ 0 & \text { if } a b \notin E(G) \text { and } a \neq b, \\ -\sum_{i=1, i \neq a}^{t} h(a i) & \text { if } a=b .\end{cases}
$$

Clearly $M(h)$ is symmetric, and each row and column has a sum equal to zero. We denote the rank of $M(h)$ by $r(h)$. We note that since the sum of its rows is zero the matrix $M(h)$ is singular, and hence $r(h)<t$.

Lemma 6. Let $G$ be a graph with $V(G)=\{1, \ldots t\}$, let $h$ be a 3 -colouring of the edges of $G$, and let $r=r(h)$. Then

$$
\rho\left(h, J_{s}\right)=(-1)^{r} 3^{-r(2 s+1)} .
$$

Proof. Let $M=M(h) . \rho\left(h, J_{s}\right)$ is the average over all functions $f$ from $V(G)$ to $G F(3)^{m}$ of the quantity

$$
\prod_{\substack{a<b \\ a b \in E(G)}}\left[\zeta_{3}^{q(f(a)-f(b))}\right]^{M_{a b}}=\zeta_{3}^{Q^{*}(f)},
$$

where $Q^{*}(f)=\sum_{a<b, a b \in E(G)} M_{a b} q(f(a)-f(b))$. For each $a \in V(G)$ we regard $f(a)$ as an $m$-tuple of variables, $\left(x_{1, a}, x_{2, a}, \ldots, x_{m, a}\right)$. Now we regard $Q^{*}(f)$ as a quadratic form in the $m t$ variables $x_{i, a}$ where $1 \leq i \leq m$ and $a \in V(G)$. In this new formulation we need to average the expression $\zeta_{3}^{Q^{*}(f)}$ over all assignments of values in $G F(3)$ to the variables $x_{i, a}$, and we will do this by diagonalising $Q^{*}(f)$.

Let $A$ be the $m \times m$ matrix with entries

$$
A_{i j}= \begin{cases}1 & \text { if } i=j \text { and } 1 \leq i \leq 2 s+2 \\ -1 & \text { if } i=j \text { and } 2 s+3 \leq i \leq m \\ 0 & \text { if } i \neq j\end{cases}
$$


This is the matrix of the quadratic form $q$, and by a routine calculation

$$
Q^{*}(f)=\sum_{a<b} M_{a b} q(f(a)-f(b))=\sum_{a<b, i} M_{a b} A_{i i}\left(x_{i, a}^{2}+x_{i, b}^{2}-2 x_{i, a} x_{i, b}\right) .
$$

Let $1 \leq p \leq t$ and $1 \leq i \leq m$. The coefficient of $x_{i, p}^{2}$ in $Q^{*}(f)$ is $\sum_{a \neq p} A_{i i} M_{a p}$. By the definition of $M, \sum_{a \neq p} M_{a p}=-M_{p p}$, and so

$$
Q^{*}(f)=-\sum_{a, i} M_{a a} A_{i i} x_{i, a}^{2}-2 \sum_{a<b, i} M_{a b} A_{i i} x_{i, a} x_{i, b} .
$$

The matrix $M \otimes A$ is the $m t \times m t$ matrix constructed by assigning $M_{a b} A$ as the $(a, b)$ entry of a $t \times t$ matrix with $m \times m$ matrices as entries. The $(i, j)$ entry of the $(a, b)$ entry is $M_{a b} A_{i j}$.

Let $P=M \otimes A$ and

$$
\bar{x}=\left(x_{1,1}, x_{2,1}, \ldots, x_{n, 1}, \ldots, x_{1, t}, x_{2, t}, \ldots, x_{m, t}\right) .
$$

An easy calculation shows that $Q^{*}(f)=-\bar{x} P \bar{x}^{\top}$.

Since the field $G F(3)$ has characteristic 3, by a standard result about quadratic forms there exists an invertible $t \times t$ matrix $C$ such that $C M C^{\top}$ is diagonal. Let $D=C M C^{\top}$, then $\operatorname{rank}(D)=\operatorname{rank}(M)=r$ by a standard argument from linear algebra. Since $D$ is diagonal, $r$ is equal to the number of nonzero entries in $D$. Let $r_{+}$be the number of 1 's in $D$, from which it follows that $r-r_{+}$is the number of -1 's. We may assume that

$$
D_{a a}= \begin{cases}1 & \text { if } 1 \leq a \leq r_{+} \\ -1 & \text { if } r_{+}+1 \leq a \leq r, \\ 0 & \text { if } r+1 \leq a \leq m\end{cases}
$$

Let $\bar{C}=C \otimes I$, where $I$ is the $m \times m$ identity matrix. By a routine calculation $\bar{C} P \bar{C}^{\top}=C M C^{\top} \otimes I A I^{\top}=D \otimes A$. The matrix $D \otimes A$ is diagonal, so we have diagonalised the form $Q^{*}(f)$; if we define a new vector of variables $\bar{y}$ such that $\bar{x}=\bar{y} \bar{C}$, then $Q^{*}(f)=-\bar{x} P \bar{x}^{\top}=-\bar{y}(D \otimes A) \bar{y}^{\top}$.

We now return to the problem of averaging $\zeta_{3}^{Q^{*}(f)}$ over all functions from $V(G)$ to $G F(3)^{m}$, or equivalently averaging $\zeta_{3}^{Q^{*}(f)}$ over all $\bar{x} \in G F(3)^{m t}$.

$$
\sum_{f: V(G) \rightarrow G F(3)^{m}} \zeta_{3}^{Q^{*}(f)}=\sum_{\bar{x} \in G F(3)^{m t}} \zeta_{3}^{-\bar{x} P \bar{x}^{\top}}=\sum_{\bar{y} \in G F(3)^{m t}} \zeta_{3}^{-\bar{y}(D \otimes A) \bar{y}^{\top}},
$$

and since $D \otimes A$ is diagonal

$$
\sum_{\bar{y} \in G F(3)^{m t}} \zeta_{3}^{-\bar{y}(D \otimes A) \bar{y}^{\top}}=\sum_{\bar{y} \in G F(3)^{m t}} \zeta_{3}^{-\sum_{a, i} D_{a a} A_{i i} y_{i, a}^{2}} .
$$


Clearly

$$
D_{a a} A_{i i}= \begin{cases}1 \quad & \text { if } 1 \leq i \leq 2 s+2 \text { and } 1 \leq a \leq r_{+} \\ & \text {or } 2 s+3 \leq i \leq m \text { and } r_{+}+1 \leq a \leq r \\ -1 \quad & \text { if } 1 \leq i \leq 2 s+2 \text { and } r_{+}+1 \leq a \leq r \\ & \text { or } 2 s+3 \leq i \leq m \text { and } 1 \leq a \leq r_{+}, \\ 0 \quad & \text { otherwise. }\end{cases}
$$

It follows that if we let $\alpha=2\left(r s+r-r_{+}\right)$and $\beta=2\left(r s+r_{+}\right)$, then the expression $-\sum_{a, i} D_{a a} A_{i i} y_{i, a}^{2}$ contains $\alpha$ terms with coefficient $1, \beta$ terms with coefficient -1 and $m t-(\alpha+\beta)$ terms with coefficient zero.

We reorder and relabel the $m t$ variables $y_{i, a}$ as $z_{1}, \ldots, z_{m t}$ so that

$$
-\sum_{a, i} D_{a a} A_{i i} y_{i, a}^{2}=z_{1}^{2}+z_{2}^{2}+\cdots+z_{\alpha}^{2}-z_{\alpha+1}^{2}-z_{\alpha+2}^{2}-\cdots-z_{\alpha+\beta}^{2} .
$$

Noting that the right-hand side depends only on the variables $z_{1}, \ldots z_{\alpha+\beta}$, we get

$$
\begin{aligned}
& \sum_{f: V(G) \rightarrow G F(3)^{m}} \zeta_{3}^{Q^{*}(f)} \\
& =3^{m t-(\alpha+\beta)} \sum_{z_{1}, \ldots, z_{\alpha+\beta} \in G F(3)} \zeta_{3}^{z_{1}^{2}+z_{2}^{2}+\cdots+z_{\alpha}^{2}-z_{\alpha+1}^{2}-z_{\alpha+2}^{2}-\cdots-z_{\alpha+\beta}^{2}} .
\end{aligned}
$$

Since $\zeta_{3}^{-1}=\zeta_{3}^{2}$, we can rewrite the right-hand side as

$$
3^{m t-(\alpha+\beta)} \sum_{z_{1}, \ldots, z_{\alpha+\beta} \in G F(3)} \zeta_{3}^{z_{1}^{2}} \zeta_{3}^{z_{2}^{2}} \cdots \zeta_{3}^{z_{\alpha}^{2}} \zeta_{3}^{2 z_{\alpha+1}^{2}} \zeta_{3}^{2 z_{\alpha+2}^{2}} \cdots \zeta_{3}^{2 z_{\alpha+\beta}^{2}}
$$

This sum is the product of $\alpha$ terms of the form $1+\zeta_{3}+\zeta_{3}^{4}$ and $\beta$ terms of the form $1+\zeta_{3}^{2}+\zeta_{3}^{8}$, so using $\zeta_{3}^{3}=1$ we get

$$
\sum_{f: V(G) \rightarrow G F(3)^{m}} \zeta_{3}^{Q^{*}(f)}=3^{m t-(\alpha+\beta)}\left(1+2 \zeta_{3}\right)^{\alpha}\left(1+2 \zeta_{3}^{2}\right)^{\beta} .
$$

Since $1+2 \zeta_{3}=\imath \sqrt{3}, 1+2 \zeta_{3}^{2}=-\imath \sqrt{3}$, and $\rho\left(h, J_{s}\right)$ is the average of $\zeta_{3}^{Q^{*}(f)}$ over the $3^{m t}$ possible values for $f$, we see that $\rho\left(h, J_{s}\right)=\frac{(-1)^{\beta}(\imath \sqrt{3})^{\alpha+\beta}}{3^{\alpha+\beta}}$. Since $\beta$ is even, $(-1)^{\beta}=1$. Because $\alpha+\beta=2 r(2 s+1)$,

$$
\rho\left(h, J_{s}\right)=(\imath)^{2 r(2 s+1)}\left(\frac{\sqrt{3}}{3}\right)^{2 r(2 s+1)}=(-1)^{r} 3^{-r(2 s+1)} .
$$


We recall from Definition 7 that $B_{3}(G)$ is the set of balanced 3-colourings of the edges of $G$. Since we are now considering 3 -colourings as taking values in $G F(3), B_{3}(G)$ is the set of colourings where the sum of the colours of the edges is equal to zero.

Definition 11. Let $G$ be a graph with $V(G)=\{1, \ldots t\}$. For each $i$ such that $0 \leq i \leq t-1, R_{i}(G)$ is the number of $h \in B_{3}(G)$ such that $r(h)=i$.

Lemma 7. Let $G$ be a graph with $V(G)=\{1, \ldots t\}$. If $V(G)$ contains a triangle, then $R_{0}(G)=1$, and $R_{1}(G)>0$.

Proof. Let $h$ be the colouring in which every edge gets colour 0 . Then $M(h)$ is the $t \times t$ zero matrix, which implies $r(h)=0$. Since the zero matrix is the only $t \times t$ matrix with $\operatorname{rank} 0, R_{0}(G)=1$.

Relabelling the vertices if necessary, we may assume that vertices 1,2 and 3 form a triangle in $G$. Let $h$ be the colouring in which the edges 12, 13, and 23 are assigned colour 1 and the remaining edges are assigned colour 0 . Then $h$ is balanced, and $M(h)$ is the $t \times t$ matrix

$$
\left[\begin{array}{ll}
1_{3 \times 3} & 0_{3 \times t-3} \\
0_{t-3 \times 3} & 0_{t-3 \times t-3}
\end{array}\right]
$$

where $1_{3 \times 3}$ is a $3 \times 3$ matrix with all entries equal to 1 , and $0_{a \times b}$ is a $a \times b$ matrix with all entries equal to 0 . Therefore $r(h)=1$, which implies that $R_{1}(G)>0$.

It is not hard to see that $R_{1}(G)>0$ if and only if $G$ contains a triangle.

Theorem 1. Let $G$ be a graph which contains a triangle. Then $G$ is 3-uncommon.

Proof. Let $G$ have $t$ vertices, so that without loss of generality $V(G)=$ $\{1, \ldots, t\}$. Let $R_{i}=R_{i}(G)$ for $i<t$, and let $B=B_{3}(G)$. By Lemma 6

$$
\Psi\left(G, J_{s}\right)=\sum_{h \in B} \rho\left(h, J_{s}\right)=\sum_{h \in B}(-1)^{r(h)} 3^{-r(h)(2 s+1)}=\sum_{j=0}^{t-1}(-1)^{j} 3^{-j(2 s+1)} R_{j} .
$$

For large $s$ the leading terms $1-\frac{R_{1}}{3^{(2 s+1)}}$ will dominate this sum. Since $R_{1}>0$ there exists an $s$ such that $\Psi\left(G, J_{s}\right)<1$. It follows from Lemma 5 that $G$ is 3 -uncommon. 


\section{Conclusion}

We have shown that there is a sequence of graph colourings $J_{s}$ which witnesses that every $G$ containing $K_{3}$ is 3 -uncommon. Note that the colouring $J_{s}$ that we constructed is $T_{1}^{\otimes(2 s+2)} \otimes T_{2}^{\otimes(2 s)}$, where $T_{i}$ is the edge colouring of $K_{3}$ in which all the edges get colour $i$.

We finish with some open questions.

1. Can the arguments of this paper be pushed further to show that larger classes of non-bipartite graphs are 3-uncommon? In particular, is every non-bipartite graph 3 -uncommon?

2. We have a very simple description of $J_{s}$ in terms of the colourings $T_{i}$. It is not hard to see that $\rho\left(h, T_{i}\right)$ can be computed recursively by a contraction-deletion formula. Can these facts be used to give a simpler proof of Theorem 1?

\section{Acknowledgements}

This paper is a revised version of a chapter from the second author's PhD thesis [9], written under the joint direction of the first author and John Mackey. We would like to thank John Mackey for many helpful discussions.

\section{References}

[1] S. Burr and V. Rosta. On the Ramsey multiplicities of graphs-problems and recent results. Journal of Graph Theory, 4(4):347-361, 1980. MR0595601

[2] P. Erdős. On the number of complete subgraphs contained in certain graphs. Magyar Tud. Akad. Mat. Kutató Int. Közl., 7:459-464, 1962. MR0151956

[3] A. Goodman. On sets of acquaintances and strangers at any party. The American Mathematical Monthly, 66:778-783, 1959. MR0107610

[4] C. Jagger. Colourings and contractions of graphs. PhD thesis, Cambridge University, 1995.

[5] C. Jagger, P. Šťovíček, and A. Thomason. Multiplicities of subgraphs. Combinatorica, 16(1):123-141, 1996. MR1394515

[6] A Sidorenko. A correlation inequality for bipartite graphs. Graphs and Combinatorics, 9:201-204, 1993. MR1225933 
[7] A. Thomason. A disproof of a conjecture of Erdős in Ramsey theory. Journal of the London Mathematical Society, 39(2):246-255, 1989. MR0991659

[8] A. Thomason. Graph products and monochromatic multiplicities. Combinatorica, 17(1):125-134, 1997. MR1466580

[9] M. Young. Triangle Problems in Extremal Graph Theory. PhD thesis, Carnegie Mellon University, 2008. MR2711661

James Cummings

Department of Mathematical Sciences

Carnegie Mellon University

PitTSBURGH PA 15213-3890

USA

E-mail address: jcumming@andrew.cmu.edu

Michael Young

Department of Mathematics

Iowa State University

AMES IA 50011

USA

E-mail address: myoung@iastate.edu

Received September 29, 2009 